\title{
Importancia de los hijos en la vejez y cambios en el comportamiento reproductivo (Estudio en tres comunidades rurales de México)*
}

\author{
Elena Zúñiga $\mathbf{H} . * *$ \\ Daniel Hernández F.***
}

En este trabajo se analiza la importancia de los hijos para el mantenimiento de la población de la tercera edad que reside en localidades rurales. El estudio se enmarca en el interés más amplio de revisar las hipótesis relativas a la transición de la fecundidad. El ejercicio que aquí se presenta incluye la revisión de la opinión de las personas entrevistadas sobre la reducción del tamaño de la familia y el control deliberado de los nacimientos y su relación con la percepción que ellos tienen del valor de los hijos.

Los datos se recopilaron en el estudio microregional sobre los " $\mathrm{De}$ terminantes del uso de métodos anticonceptivos en el área rural de México". La información corresponde a la región de Temascaltepec, compuesta por tres localidades con menos de 1500 habitantes, en el Estado de México. Se emplean datos obtenidos mediante cuestionarios estructurados e información de historias de vida de una submuestra aleatoria de parejas o personas de 60 años o más.

Se describe la dinámica de las unidades domésticas y el tipo y estructura de los hogares en los que residían las personas de 60 años o más. Se analiza la percepción de los padres sobre el valor de los hijos, registrándose un fuerte contenido económico o de asistencia instrumental, que va cambiando conforme los hijos van transcurriendo por su ciclo de vida individual. Para identificar las formas de manutención de las personas de mayor edad, se revisó su participación en la actividad económica, observándose que los individuos tienen que continuar trabajando para su manutención hasta edades avanzadas.

Con respecto a las condiciones en que los hijos apoyan a sus padres en la vejez, se indagó sobre la ayuda económica que cada uno de ellos brindaba. Finalmente se presentan las preferencias sobre el tamaño de la familia de la población de 60 años o más y las razones expresadas por las mujeres sobre la conveniencia de controlar la fecundidad y reducir el tamaño de la familia, en que incluyen principalmente tres tipos de costos: el costo económico que representó la manutención y el cuidado de los hijos; el costo emocional que implicó su crianza y en cierta forma la reproducción de la pobreza, y el costo en salud derivado de los embarazos y partos.

* El estudio original del que se desprende este trabajo se realizó bajo el auspicio de la Organización Mundial de la Salud.

** Centro de Estudios en Población y Salud, SSA

*** Jefatura de Salud Reproductiva y Materno Infantil, IMss. 
Introducción

En este trabajo se hace un análisis de la importancia de los hijos para el mantenimiento de la población de la tercera edad, que reside en localidades rurales, principalmente se considera la percepción que tiene esta población sobre la ayuda económica que le brindan y brindaron sus hijos a lo largo de su vida y algunos indicadores sobre el aporte económico de cada hijo sobreviviente.

El objetivo de abordar esta problemática se enmarca en el interés más amplio de revisar las hipótesis relativas a la transición de la fecundidad, sobre todo la que plantea que mientras los hijos signifiquen un valor económico para sus padres y la única seguridad en la vejez, la fecundidad se mantendrá elevada.

Desde esta perspectiva, el ejercicio que aquí se presenta incluye la revisión de la opinión de las personas entrevistadas sobre la reducción del tamaño de la familia y el control deliberado de los nacimientos y su relación con la percepción que ellos tienen del valor de los hijos.

Se tomó la decisión de definir la "vejez" con un criterio de edad, de manera que se incluye a toda la población con 60 años o más, lo que se desprendió de considerar la esperanza de vida al nacer que se estimó de 65 años en el medio rural, en 1985.

Esta definición presentó el problema de incluir a una población con gran variedad en sus capacidades físicas, lo que, aunado a que en el contexto rural la participación económica de los individuos continúa hasta edades avanzadas, hace que prácticamente no sea factible ubicar un punto de corte a partir del cual se depende por completo del apoyo de los hijos. Además las personas definidas como "viejas" bajo este criterio, pueden estar atravesando etapas distintas en el ciclo de vida de manera que algunos de los entrevistados tienen aún hijos pequeños en el hogar, mientras que otros solo tienen hijos mayores de 30 años.

El trabajo se basa en la información recopilada en el estudio "Determinantes del uso de métodos anticonceptivos en el área rural de México. Estudio comparativo microregional", cuyo trabajo de campo se realizó de septiembre a diciembre de 1985.

Este trabajo se realizó con la información correspondiente a la región de Temascaltepec, compuesta por tres localidades de este municipio, con menos de 1500 habitantes cada una, ubicadas en la parte sur-occidental del Estado de México. 


\section{Metodología y fuentes de datos}

Se utilizaron dos técnicas para la obtención de información. Por un lado, se emplearon cuestionarios estructurados que se aplicaron a todos los hogares, a todas las mujeres en edad fértil unidas y a las personas o parejas de 60 años o más (al menos a uno de los miembros de la pareja). Estos cuestionarios se dirigieron a obtener datos sobre las características de las familias, en términos de la organización del trabajo familiar y de los recursos disponibles, así como de la conducta reproductiva de las mujeres en edad fértil.

El módulo para personas o parejas de 60 años o más, de este censo de localidad, incluye una tabla en la que se pide listar a todos los hijos nacidos vivos que haya tenido y para cada uno de ellos: su status de sobrevivencia, edad a la que falleció, su lugar de residencia actual, fecha en la que salió del hogar, fecha en la que salió de la comunidad, estado civil, escolaridad, si envía dinero o alimentos al hogar y la regularidad del envío. Con esta información se pretende identificar la proporción de hogares que reciben ayuda y algunas características de los hijos que la otorgan. El cuestionario de hogar, por su parte, permite identificar las estructuras de parentesco y composición de las familias que caracterizan a las unidades domésticas de la población de la tercera edad.

Por otro lado, con el objeto de recuperar la percepción de la población sobre las implicaciones y beneficios de tener hijos, se recopilaron las historias de vida de una submuestra de parejas o personas de 60 años o más. Cabe hacer notar que la selección de estas submuestras fue aleatoria, a partir de un ordenamiento progresivo de las personas según su edad, y esto con la sola idea de asegurar que el investigador no seleccionara a los informantes con los que ya había establecido un contacto o eran los más fáciles de entrevistar. Sobre todo entre la población mayor es muy frecuente que se nieguen a dar la entrevista por pena o temor, por lo que la selección aleatoria requirió una labor de acercamiento de los investigadores hacia esta población.

Se entrevistaron 15 mujeres en edad fértil unidas y cinco personas mayores de 60 años en cada una de las tres localidades consideradas del municipio de Temascaltepec. Además, se completó la información sobre las comunidades mediante entrevistas a personal de salud y líderes comunitarios. En total, el universo quedó conformado por 431 hogares, 299 mujeres en edad fértil unidas y 89 personas o parejas mayores de 60 años. De estos últimos se levantaron 15 entrevistas en profundidad.

El trabajo de campo fue realizado por seis encuestadoras que aplicaron los cuestionarios cerrados del censo de cada localidad, 
y tres investigadoras que realizaron las entrevistas en profundidad y el estudio de comunidad, permaneciendo para ello en la localidad por un periodo de tres meses.

\section{Características de las tres comunidades}

Las comunidades seleccionadas fueron San Mateo Almomoloa, San Miguel Oxtotilpan y Mesón Viejo. Las tres se encuentran a 42 y $35 \mathrm{~km}$ de la ciudad de Toluca, cercanas entre sí y de la carretera México-Zihuatanejo. Esta carretera fue terminada en 1965, momento en el cual se logró un cambio importante en la integración de estas comunidades con los dos centros económicos más importantes de la región: la ciudad de Toluca y la ciudad de México.

Las localidades presentan semejanzas en sus características climáticas (subhúmedo y semifrío), asentadas en lomeríos rodeados de cerros con bosques de pino, encino y ocote. A pesar de la cercanía de las comunidades, presentan diferencias acentuadas en el nivel de desarrollo económico y social alcanzado.

San Mateo Almomoloa se encuentra a $3 \mathrm{~km}$ de la carretera, es un poblado con 1154 habitantes en 165 hogares. Todas las tierras son de propiedad ejidal y comunal con un total de 3270 ha. de las cuales $20 \%$ son cultivables. El $90 \%$ de los hogares posee una parcela, de las que $80 \%$ tiene una extensión no mayor a una hectárea. El cultivo es de temporal, y se produce principalmente maíz y frijol para la autosubsistencia. Práctican también la ganadería y unos cuantos productores siembran papa y chícharo para su venta. También recolectan resina, zacatón para la producción de escobas y raspan magueyes para la producción de pulque. La actividad más importante para la obtención de dinero es la venta de su fuerza de trabajo, principalmente en la actividad agrícola de la zona. Asimismo, se acostumbra la emigración temporal para trabajar como albañiles en las ciudades. La mayoría de la gente vive en condiciones muy precarias y es la comunidad más pobre de las tres incluidas en este estudio.

San Miguel Oxtotilpan se ubica a $4 \mathrm{~km}$ de la carretera MéxicoZihuatanejo, a la que se llega por camino de terracería terminado en 1975. Cuenta con 912 habitantes en 165 hogares. Las formas de tenencia de la tierra son ejidal, privada y comunal. Casi la totalidad de los hogares cuentan con tierra, de los cuales poco más de la mitad posee una hectárea, $40 \%$ dos o tres y el resto entre 5 y 17 hectáreas. La actividad principal es la agricultura de temporal y de riego. Producen maíz, frijol y habas que se destinan al consumo familiar. Los productores que tienen más recursos siembran 
avena, que en parte se comercializa y en parte se destina al consumo de los animales, y papa destinada al mercado. Al igual que en San Mateo, la población con menos recursos se asalaria, ya sea en el trabajo agrícola o en las ciudades como albañiles o en el trabajo doméstico. Hay explotación forestal, que brinda trabajo a un número reducido de personas de la comunidad, pero que genera un ingreso complementario a los comuneros. Debido a la existencia de tierras de riego y en general de una relativa mejor calidad de las tierras, San Miguel presenta mejores condiciones socioeconómicas que San Mateo.

Mesón Viejo es un pequeño poblado, ubicado a un kilómetro de la carretera México-Zihuatanejo, que cuenta con 403 habitantes y 71 hogares. El $75 \%$ dispone de tierras en propiedad ejidal y (o) privada. Actualmente toda la tierra de cultivo tiene acceso al sistema de riego por canales. El $60 \%$ de los productores cuenta con una hectárea, una tercera parte entre 2 y 3 y una minoría entre 4 y 7 hectáreas, más otras que arriendan fuera de la localidad. Los pobladores son agricultores, producen maíz y frijol para el autoconsumo, pero son principalmente productores de papa y avena para el mercado. Crían animales como borregos y aves de corral. Las personas que no tienen tierra o tienen poca se "alquilan" como peones de campo y también hay quienes emigran temporalmente a las ciudades en busca de trabajo, principalmente los hijos solteros.

En el cuadro 1 se puede apreciar la enorme ventaja en las condiciones de vida de los residentes de Mesón Viejo con respecto a los pobladores de las dos comunidades vecinas. No sólo la totalidad de la tierra es de riego, lo que permite la agricultura comercial, sino que además tienen acceso a mayores extensiones de tierra y mayores insumos productivos que a las otras dos localidades, especialmente con respecto a San Mateo Almomoloa.

Las viviendas de Mesón Viejo cuentan con mejores materiales de construcción y servicios. Así, hay menos viviendas con piso de tierra ( $24 \%$ contra $80 \%$ en San Mateo); el material de las paredes es adobe o tabique en $80 \%$ contra $42 \%$ de las viviendas de San Mateo; la gran mayoría dispone de agua entubada y luz eléctrica contra una minoría en San Mateo y, en general, ocurre lo mismo con el resto de los indicadores. San Miguel Oxtotilpan presenta las condiciones intermedias de las tres comunidades.

En la historia de las comunidades se encuentra la explicación de este desarrollo desigual. Mesón Viejo es un asentamiento relativamente joven. A mediados del siglo XIX comenzaron a llegar las primeras familias oriundas de San Miguel Oxtotilpan. Donde ahora se encuentra la calle principal fue un camino por el que se 
CUADRO 1

Características socioeconómicas de los hogares

\begin{tabular}{lccrr}
\hline & & & Mesón & \\
& Sn. Mateo & Sn. Miguel & Viejo & Total \\
\hline Tiene parcela & 90.8 & 92.7 & 74.6 & 88.9 \\
1 ha, de cultivo o menos & 80.2 & 54.2 & 59.6 & 65.7 \\
Usa tractor & 0.6 & 10.4 & 13.2 & 6.3 \\
Usa fertilizantes químicos & 94.4 & 82.5 & 92.5 & 89.3 \\
Usa insecticidas & 1.7 & 33.3 & 86.8 & 26.1 \\
& & & & \\
Material pisos tierra & 80.5 & 47.3 & 23.9 & 58.5 \\
Material paredes adobe y tabique & 42.1 & 63.1 & 80.2 & 56.4 \\
Material techo teja y concreto & 1.5 & $\mathbf{9 . 1}$ & 42.2 & 11.2 \\
Agua entubada & 13.8 & 33.9 & 73.2 & 31.3 \\
Luz eléctrica & 45.1 & 80.0 & 85.9 & 65.2 \\
1 a 2 cuartos para dormir & 76.9 & 63.7 & 36.6 & 65.2 \\
Sin drenaje & 27.7 & 69.1 & 43.7 & 46.2 \\
Combustible cocina leña & 97.4 & 94.5 & 71.8 & $\mathbf{9 2 . 1}$ \\
& & & & \\
Tiene radio & 56.9 & 66.1 & 63.4 & 61.5 \\
Tiene TV & 4.6 & 13.9 & 8.5 & 8.8 \\
Tiene refrigerador & 0.5 & 1.8 & 14.1 & 3.2 \\
Tiene automóvil & 2.1 & 2.4 & 8.5 & 3.2 \\
\hline
\end{tabular}

Fuente: estudio "Determinantes del uso de métodos anticonceptivos en las áreas rurales". Cuestionario de hogar.

llegaba a tierra caliente (Ciudad Altamirano y a los límites de los estados de Michoacán, Guerrerro y Distrito Federal) y a la ciudad de Toluca. Por este camino transitaban los entonces "arrieros" y "aventureros"; los primeros vendían productos forestales de los ranchos y los segundos llevaban a vender ganado de engorda y puercos. Los primeros pobladores comenzaron a cultivar estas tierras y posteriormente a construir sus casas, a fin de estar cerca del camino para vender y comprar productos. El nombre de Mesón Viejo resultó de la costumbre, ya que a orillas del camino había una casa abandonada a la que se conocía con este nombre que utilizaban para dormir los viajeros.

Estos pobladores eran ladinos. En cambio, en San Mateo predomina población indígena (nahuas) y en San Miguel, aunque son minoría, también constituyen un grupo importante. Parece que la población de Mesón Viejo tenía una relación favorable con la hacienda La Gavia, a la cual pertenecía la tierra de las tres comunidades hasta antes del reparto agrario, ocurrido en el primer quinquenio de la década de 1930. Antes del reparto, la hacienda regaló tierra a algunos 
de los campesinos que trabajaban en ella, con lo que se inició la pequeña propiedad en la región. Desde principios del siglo, en las tierras de Mesón Viejo, se construyó un canal que sirvió para regar gran parte de la tierra cultivable al tiempo que se sembraba maíz y trigo.

Con la construcción de la carretera México-Zihuatanejo, los productores de Mesón Viejo iniciaron el cultivo de la papa en sustitución del trigo, con lo cual obtuvieron mayores rendimientos. Los primeros años fueron de gran auge, alcanzándose el climax en 1981. En estos años los cambios ocurrieron rápidamente, los productores más exitosos (cerca de 20 de los 70 hogares), adquirieron camionetas y camiones, de manera que en la actualidad la comercialización la realizan directamente en la Central de Abastos de la ciudad de México. Las mujeres y los jóvenes se acercaron a las ciudades no sólo para satisfacer el consumo, sino también para asistir a fiestas y centros de recreación.

En los años anteriores al estudio se vivió en la zona una crisis económica derivada de la caída de los precios de la papa. Los productores de otras comunidades fueron incorporando superficies a este cultivo, de manera que la competencia se incrementó y se redujeron los precios, lo que unido al desgaste del suelo ha provocado pérdidas considerables por lo que muchos productores han dejado de sembrarla.

San Miguel Oxtotilpan, también se benefició del auge de la papa, favorecido por el acceso al sistema de riego de gran parte de su tierra cultivable.

San Mateo quedó al margen de este proceso, resultado de la mala calidad de la tierra y, probablemente, discriminado desde la época de la hacienda por ser población predominantemente indígena.

Los servicios de luz y agua potable se instalaron en la región por los mismos años. La electrificación de las tres comunidades ocurrió en el año de 1961, pero sólo en las viviendas concentradas a orillas del camino principal. Por ello, la mayoría de las viviendas cuenta con este servicio en San Miguel y Mesón y sólo menos de la mitad en San Mateo, que es un poblado de asentamiento disperso. El sistema de agua potable de llaves públicas se instaló en 1970, y en 1974 se hicieron las primeras tomas domiciliarias en Mesón y San Miguel, mientras que en San Mateo fue en 1976.

Los servicios de educación primaria completa tenían entre 15 y 20 años de existir en cada una de las comunidades cuando se realizó el estudio. Desde mediados del siglo, se otorgó enseñanza hasta tercero de primaria; más tarde lograron que se impartieran los seis años: en 1961 en San Mateo, en 1965 en San Miguel y en 
1970 en Mesón Viejo. Ninguna de las comunidades cuenta con niveles superiores a la educación primaria.

Sólo en San Mateo y en San Miguel existen servicios médicos. Son Unidades Médicas Rurales del Instituto Mexicano del Seguro Social que se instalaron en 1981. Un Centro de Salud de la SSA, se instaló en un poblado cercano en 1976. Estos son los únicos servicios médicos con los que cuentan las comunidades, además de los agentes tradicionales de salud como parteras y curanderos. En Mesón Viejo no se cuenta con clínicas del sector salud pero tienen derecho de servicios de la clínica de San Miguel. En la zona no existe medicina moderna privada.

\section{Resultados}

El análisis de las historias de vida de la población mayor de 60 años de las comunidades rurales, que comprende a una generación que se encuentra prácticamente en los últimos años del ciclo de vida familiar, presenta la enorme ventaja de poder reconstruir no sólo las percepciones del valor de los hijos en el presente, sino también desde la perspectiva de las diferentes etapas del ciclo de vida por las que transcurre la familia.

Conviene detenerse a definir algunos de los conceptos que utilizaremos en esta presentación. La unidad doméstica se refiere a la unidad conformada por el conjunto de individuos que hacen vida común, comparten los gastos de la casa y se organizan, bajo la tutela de un jefe del hogar, para cubrir las funciones domésticas relativas a la reproducción cotidiana y generacional de los miembros de la unidad, así como también las actividades productivas en las que se incluye tanto la obtención de ingresos monetarios como de productos para el autoconsumo o el mercado.

Un ciclo familiar se inicia con la formación de una pareja, la cual puede incorporarse a una unidad doméstica o familia ya conformada. Por su parte, la unidad doméstica o familiar se inicia cuando alguno de los miembros de la pareja, generalmente el hombre, asume la jefatura del hogar y termina cuando ninguno de los dos miembros de la pareja continúa siendo el jefe.

Con el objeto de identificar la dinámica de las unidades domésticas, se describe a continuación el patrón general que se desprende del análisis de las historias de vida de las 15 personas entrevistadas en profundidad de las tres comunidades. Se busca retroalimentar este análisis con la información estadística que se desprende del censo de localidades (del cuestionario de hogar y del de personas de 60 años o más). 


\section{Estructura y dinámica de las unidades domésticas}

La pareja se constituye cuando el hombre se "roba" a la mujer, según la terminología de Temascaltepec. En la mayoría de los casos entrevistados las mujeres tenían entre 13 y 17 años de edad cuando se inició la unión. Si bien este "robo" se realiza bajo el acuerdo de la novia, en algunos casos la unión toma la forma de un robo en el sentido textual, por ser producto de la sumisión que caracteriza a las mujeres hacia la voluntad de su pareja, sobre todo a esas edades, y también porque en algunos casos se hizo bajo el engaño, con la complicidad de otros adultos. El matrimonio legal, y más aún el religioso, antes de la corresidencia con la pareja, es un ideal para la mujer, que pocas alcanzan. De cualquier forma, una vez que se une la pareja se recurre a un pariente para poder iniciar la vida conyugal. Por ello, prácticamente en todos los casos, la pareja inició la vida conyugal integrándose a una familia ya conformada, que generalmente corrrespondía a la de los padres o tutores del esposo.

Algunos de los entrevistados se robaron a la novia en la ciudad de México, donde ambos trabajaban. Ellos también acudieron con un pariente, ya sea que se llevaran a la mujer al pueblo de origen con los padres y ellos continuaban trabajando en la ciudad, o bien con una tía o pariente que radicara en la ciudad de México.

Esta práctica parece tener que ver más con la solidaridad de los adultos hacia los jóvenes, aunque permite que los padres cuenten con la ayuda de algún hijo incluso después de que éste se casa. Sin embargo, para la pareja significa disponer de un techo, que con los bajos salarios que se obtienen en la ciudad no logran afrontar sin el apoyo de otros familiares.

En realidad, los entrevistados asumen la corresidencia con los parientes como algo completamente esperado o lógico; en parte porque es la forma en que se legitima en términos sociales la unión de la nueva pareja y en parte porque nadie espera que sea de manera diferente. Hubo un caso en que la pareja se mudó a la casa de la madre de la esposa; el fenómeno es tan anormal que el hombre dijo de sí mismo que se "anueró" por este hecho. Los que no residieron con un pariente (dos casos) tienen la característica de haberse casado más grandes y haber juntado algún dinero para comprar el pedazo de solar en el que levantaron la casa. Cuando la pareja se independiza, la mujer trabaja casi a la par del marido en la parcela o como jornalera, lo que hace más complicado el cuidado de los hijos y los embarazos de estas mujeres.

Mientras viven en el hogar de los padres del esposo, el ingreso que éste obtiene continúa entregándolo a sus padres, en tanto 
que la mujer está sujeta a las decisiones de la suegra. La mujer, por lo general, está al margen de la toma de decisiones y manejo del gasto doméstico. Las mismas mujeres describen el trato que recibieron en el hogar de los suegros como el de una hija, por no tener ninguna ingerencia en el manejo del dinero o gasto de la casa y estar sujeta a la autoridad de los suegros.

Durante esta etapa, las parejas inician la crianza de los hijos. El primer hijo llega muy rápido y los siguientes embarazos alrededor de cada dos años. Generalmente, para antes de que el primer hijo cumpliera la edad de ocho años la pareja ya tenía entre tres y cuatro hijos.

Sin embargo, existe la tendencia a que las parejas conformen su propia unidad doméstica, sobre todo cuando los padres del esposo todavía tienen otros hijos en el hogar. La nuclearización de la familia ocurre en muy diferentes momentos, usualmente entre los 4 y 7 años después de la unión. Las causas de la nuclearización también varían, pero en muchos de los casos responde al interés de los padres más que de los hijos, y resulta de la expansión de la familia por el matrimonio de varios de ellos.

La nueva unidad doméstica que se crea al independizarse la pareja prevalece nuclear hasta que los hijos crecen y un hijo varón trae consigo a su pareja. Algunos de los hijos residirán con los padres y al final, el más chico, o el que continúa ayudando en el trabajo parcelario, se queda en el hogar hasta la muerte de ellos. En esta etapa, generalmente se busca que permanezca un hijo en el hogar para que se haga cargo de la explotación de los recursos productivos de la unidad doméstica y ayude a las personas mayores. Muchos de los casos de parejas o viejos que residían solos se debía a la falta de atractivo para los hijos de continuar en la comunidad y a las condiciones paternas.

\section{Estructura de los hogares de los viejos}

Con la información del censo pueden identificarse los tipos de hogares en los que residían las personas de 60 años o más al momento de la entrevista. Esta tipología de hogares se construyó con los datos de los cuestionarios de hogar. ${ }^{1}$

Las personas mayores de 60 años residen más frecuentemente en hogares extensos (definidos como aquéllos en que por lo menos un miembro no corresponde a un núcleo de padres e hijos)

1 Se define a patir de las relaciones de parentesco de los miembros del hogar respecto al jefe económico, que es la persona que mayor ingreso aporta al hogar. 
que las unidades domésticas donde no se encuentran personas en la vejez. En Temascaltepec $\mathbf{4 9 . 0 \%}$ de los hogares con personas mayores de 60 años se define como extenso, en comparación con un $23.5 \%$ de los hogares sin personas en estas edades.

Se perciben diferencias en la composición de los hogares entre las comunidades. En San Mateo, que es la comunidad más pobre de las tres, se recurre en mayor medida a la conformación de familias extensas: $53 \%$ de los hogares donde reside al menos una persona de 60 años o más es extenso. En Mesón Viejo, que constituye el contraste en cuanto al nivel socioeconómico de la población, sólo $42 \%$ de los hogares de viejos son extensos.

Los hogares nucleares constituyen casi la mitad de los de la gente mayor. De ellos, la mayoría corresponde a las unidades domésticas constituidas por el jefe y su cónyuge o sólo por el jefe ( $23 \%$ del total de los hogares de gente mayor en Temascaltepec), lo que muestra que una proporción importante de mayores de 60 años vive sola y, presumiblemente, recibe menos apoyo económico de parte de sus hijos. En esta aseveración no se está considerando la residencia contigua, en hogares independientes, lo que puede permitir apoyos menos tangibles y sin duda otros de tipo emocional que ayudan en la sobrevivencia de los viejos.

En el caso de las familias extensas, que constituyen la otra mitad de los hogares de las personas mayores, se ha podido identificar que los viejos son los jefes económicos del hogar en la mitad de los casos de las familias extensas. En la otra mitad, los viejos constituyen el componente no nuclear y son principalmente las madres o padres del jefe.

Así, del total de las personas de 60 años o más censadas en las comunidades, $73.2 \%$ son jefes o cónyuges y una quinta parte madre o padre del jefe. Entre las comunidades, se observa que en San Mateo los viejos son con menos frecuencia jefes en los hogares (53.6\%) que en Mesón Viejo o San Miguel (63.2 y 67.3\% respectivamente) lo que sugiere una mayor pérdida por parte de los viejos del control de los recursos en los hogares de mayor pobreza.

\section{Papel de los hijos en diferentes etapas del ciclo familiar}

En términos generales, puede decirse que la percepción de los padres sobre el valor de los hijos tiene un fuerte contenido económico o de asistencia instrumental, que va cambiando conforme los hijos van transcurriendo por su ciclo de vida individual.

Cuando se forma el matrimonio se sabe que los años difíciles serán aquéllos en los que sólo se cuenta con niños pequeños, pero 
que en cuanto inicien y desarrollen actividades productivas la familia verá su retribución económica. En este sentido, se percibe que en estos años los hijos sólo significan 'gasto' para sus padres.

Nos las vimos muy difícil cuando estaban chicos los niños. Al principio no podía ayudarle mucho a mi señor, pero luego que van creciendo ya me los cargaba en la espalda y le ayudaba en la cosecha. Claro que no se compara lo que podía hacer a cuando van creciendo los niños.

Cuando eran chamacos, los problemas eran para darles de comer, para mandarlos a la escuela; mi señor tenía que irse a México para trabajar, pues se necesitaba mucho trabajo para que luciera el dinero, pues todo era gasto. Cuando fueron creciendo fue menos la necesidad, pues ellos fueron ayudando.

La siguiente etapa del ciclo de vida de la familia podría definirse como aquella en que los hijos hombres empiezan a trabajar en la parcela, las mujeres comienzan a ayudar a la madre en el cuidado de los hermanos pequeños o en las labores domésticas y ambos, niños y niñas, participan en la crianza y cuidado de los animales.

También los viejos tienden a participar en el cuidado de los animales, casi como el último tipo de trabajo que realizan antes de la incapacidad laboral total o la muerte. Es como un ciclo de la familia con el que se inicia y termina la capacidad productiva de la población de estas comunidades. En este momento del ciclo se considera que los niños aportan una ayuda marginal, cuyo efecto principal es reducir la carga de trabajo doméstico (puede ubicarse este periodo cuando los niños tienen entre 8 y 14 años de edad).

Mientras los hijos están chicos sólo hacen mandaditos, como acarrear leña, en el campo ayudaban a echar la "mejora".

Ya a los 8 años empiezan a ayudar. Los niños en cosas de niños y las niñas en sus propios quehaceres, pero todos ayudan y ya por ahí de los 13 o 15 empiezan a ganar sus centavitos.

Posteriormente, los hijos alcanzan su mayor capacidad productiva, que se relaciona con aportar ingresos monetarios al hogar. Se alcanza la edad en que se puede optar por un trabajo asalariado y se emigra de la comunidad. Son pocos los casos en que este patrón no ocurrió. Los hombres y mujeres de la región de Temascaltepec migran principalmente a la ciudad de México, a trabajar como albañiles y sirvientas. En San Mateo se presentan más casos en que no hubo emigración de los hijos que en Mesón Viejo y San Miguel, en algunos de los hogares los hijos han migrado para ocuparse como obreros e inclusive para estudiar. 
Los hijos que no se van de la comunidad se ocupan como jornaleros en la agricultura y ayudan a cultivar la propia parcela. Todos los entrevistados coinciden en considerar este periodo como el de mayor ventaja económica, cuyos efectos consisten en la mejoría sustancial de la dieta familiar y en el incremento de la capacidad de ahorro expresada en la adquisición de animales o la realización de mejoras a la vivienda.

A esta edad los hijos ya son capaces de ayudar a sembrar y a cosechar, así como cultivar para otras personas.

Cuando (los hijos) ya estaban grandes, los hombres se iban con el papá a sacar raíz y las mujeres ayudaban a lavar la raíz y al quehacer de la cocina. También, tanto hombres como mujeres se alquilaban con el papá para sembrar, cosechar y manejar la yunta.

Cuando los hijos emigran de la comunidad al mercado de trabajo urbano, el monto de ingresos monetarios que incorporan al hogar alcanza la máxima cantidad que los hijos brindan a sus padres. Prácticamente no hay diferencias entre hijos del sexo femenino o masculino; ambos tienen la "obligación" de ayudar, enviando dinero al hogar. Muchas de las declaraciones hicieron referencia a que si los hijos no salen de la comunidad entonces no brindan ayuda, porque o no aportan dinero sino trabajo, o éste es muy reducido.

Esta "obligación" de ayudar a los padres aparentemente no se sustenta más que en los lazos de solidaridad y en el altruismo de hijos a padres. La "obligación" con la familia paterna dura hasta el matrimonio y sobre todo hasta que los hijos tienen a sus propios hijos.

Los hijos solteros son el verdadero sostén. Ellos como no tienen otra obligación ayudan a la casa.

Los hijos cuando solteros sí ayudan; casándose, ¿quién no da ni un centavo? Ellos ya tienen sus obligaciones.

Veía yo a las señoras con sus hijos grandes que les traían cosas y me daba harta envidia y decía cuándo será el día que a mí me ayuden mis hijos. Cuando empezaron a crecer y a irse me ayudaban bastante. Nos compramos la yunta cuando mi hijo trabajó en el aserradero. Toda la raya era para la casa. Entonces ya no tuvimos tantas apuraciones, pudimos comer mejor, comprar algunos animales, sembrar y comprar algo de ropa. Claro, ya casados no es lo mismo, ya no se les puede exigir.

La interrupción de la ayuda económica a los padres en realidad ocurre cuando los hijos constituyen su propia unidad doméstica o se separan de la unidad paterna. Cuando el hijo lleva a su 
pareja a residir al hogar paterno, el ingreso monetario continúa siendo administrado por la madre y no por la esposa o pareja.

Cuando los hijos tienen que mantenerse por separado, los padres reducen sus expectativas de ser ayudados. Las hijas porque pasan a depender de las decisiones del marido y los hombres porque deben responder primero a su pareja y sus hijos.

Finalmente, durante la vejez prevalece la expectativa que los hijos (o al menos uno de ellos) se harán cargo de los padres cuando estos ya no puedan trabajar. Sin embargo, la satisfacción de esta expectativa dependerá tanto de la voluntad de los hijos como de si cuentan con las posibilidades económicas para ayudarlos (sobre todo si viven fuera de la comunidad).

En general, una vez que los hijos se han independizado la ayuda que brindan a sus padres depende de su voluntad. En palabras de los viejos, dependerá de lo "buenos" o "malos" que resulten ser.

\section{Formas de manutención de las personas mayores}

Una manera de identificar las formas de manutención de las personas de mayor edad es mediante su participación en la actividad económica. Tanto las entrevistas en profundidad como el censo indican una elevada continuidad en el trabajo por parte de esta población. Alrededor de $70 \%$ de estos individuos realiza una actividad económica. No obstante que en las tres comunidades estos porcentajes son elevados, en San Mateo y San Miguel alcanza a $80 \%$ mientras que en Mesón Viejo es de sólo 62 por ciento. La mayor permanencia en el trabajo de este tipo de miembros del hogar en las primeras dos comunidades se desprende de la mayor proporción de mujeres que continúan trabajando (72 y $68.4 \%$ en San Mateo y San Miguel, respectivamente, contra $38.5 \%$ en Mesón Viejo), en tanto que $90 \%$ de los varones de mayor edad trabajan en las tres comunidades.

Es claro que dentro del grupo de mayores de 60 años se encuentran personas de muy diversas edades. Para identificar la edad en que estos individuos dejan de trabajar, se obtuvieron porcentajes de participación económica por grupos etáreos más pequeños. Los resultados indicaron que la participación económica se mantiene en niveles elevados (por encima de $75 \%$ ) hasta antes de los $\mathbf{8 0}$ años en las tres comunidades, para el caso de hombres y mujeres, con excepción de Mesón Viejo, en el que las mujeres a partir de los 70 años participan en proporciones muy reducidas.

Resulta claro que en estas comunidades rurales los individuos tienen que continuar trabajando para su manutención hasta eda- 
CUADRO 2

Porcentaje de personas de 60 años y más que continúa trabajando en actividad remunerada o en el recurso productivo del hogar según grupos de edad y región

\begin{tabular}{lrrrrrrrrr}
\hline & \multicolumn{2}{c}{ San Mateo } & \multicolumn{3}{c}{ San Miguel } & \multicolumn{3}{c}{ Mesón Viejo } & \multicolumn{2}{c}{ Total } \\
& \multicolumn{1}{c}{$\%$} & $n$ & $\%$ & $n$ & $\%$ & \multicolumn{1}{c}{$n$} & $\%$ & $n$ \\
\hline $60-69$ & 90.9 & 11 & 90.2 & 41 & 90 & 10 & 90.3 & 62 \\
$70-79$ & 81.8 & 11 & 87.0 & 23 & 50 & 10 & 77.3 & 44 \\
80 y más 62.5 & 8 & 46.7 & 15 & 25 & 5 & 48.1 & 27 \\
\hline
\end{tabular}

Fuente: estudio "Determinantes del Uso de Métodos Anticonceptivos en las Áreas Rurales". Cuestionario de Hogar

des avanzadas, lo que hace pensar que el apoyo instrumental de los hijos en la vejez no resulta suficiente para permitir que los individuos puedan dejar de trabajar sino hasta que se encuentren incapacitados para seguir trabajando.

En las entrevistas en profundidad se confirma que la gran mayoría de estas personas continúa realizando labores productivas. Inclusive mujeres de San Mateo y San Miguel, mayores de 70 años, continúan trabajando como jornaleras cuando consiguen quién las ocupe. Esto es independiente de si tienen hijos o no en el hogar. En Mesón Viejo es menos frecuente la participación económica de las mujeres, por lo que cuando enviudan o quedan solas tienden en mayor medida a reclutarse en la unidad doméstica de alguno de sus hijos o a cederles la jefatura de la suya.

La parcela es la actividad que más ocupa a estos individuos ( $78 \%$ en San Mateo y San Miguel y $54 \%$ en Mesón Viejo del total de personas de 60 años y más). La segunda actividad en importancia es el negocio familiar (alrededor de $20 \%$ de los viejos de las tres comunidades) que se refiere a la cría de borregos y la venta de misceláneos, sobre todo refrescos, cervezas y pulque. En San Mateo, casi una cuarta parte de estas personas trabaja como jornaleros, porcentaje muy superior al declarado en las otras dos comunidades.

La explotación de la parcela no puede ser realizada totalmente con el trabajo de los viejos, sobre todo de los mayores de 70 años. Se requiere la ayuda de otros miembros de la familia e inclusive de trabajadores asalariados. En San Mateo y San Miguel es muy frecuente establecer convenios con otros productores, de manera que los viejos prestan la tierra a cambio de parte del producto parcelario. También se acostumbra, tanto para la siembra como para la cosecha, la "vuelta de peón", que significa que un grupo de personas, por lo general parientes, se junten para realizar el trabajo de sus respectivas parcelas y evitar la contratación de tra- 
bajadores. Sin embargo, para los viejos lo más deseable es que alguno de los hijos o de los yernos, se haga cargo de estas labores. Por ello, se busca que al menos uno continúe en el hogar bajo la promesa de heredar la tierra y la casa o bien cediéndoselas en vida, lo que es más frecuente en la comunidad de San Mateo.

La mayoría de los hogares de los viejos cuenta con hijos residiendo en el hogar (cerca de 70\% de los hogares de San Mateo y de Mesón Viejo y 48\% en San Miguel). Puede decirse que, virtualmente en todos ellos, las personas mayores reciben algún tipo de ayuda por parte de sus hijos.

También gran parte de los hogares tienen hijos residiendo fuera de la localidad, principalmente en la ciudad de México. En San Miguel y Mesón Viejo esta característica la presenta casi el $100 \%$ de los hogares, mientras que en San Mateo sólo 60 por ciento. Los hijos migrantes llegan a representar una gran ayuda para sus padres. En los tiempos críticos del cultivo envían dinero para cubrir el gasto de la parcela o bien regresan para realizar estas labores. También los hijos migrantes, principalmente los solteros, son pilares para el mantenimiento cotidiano de los viejos: enviándoles dinero regularmente o alimentos y vestido. Asimismo, estos hijos facilitan que los padres reciban atención médica para enfermedades crónicas y degenerativas.

Entre las comunidades se presentan diferencias en cuanto a este patrón. En San Mateo que es la comunidad con menos emigración, los que lo hacen también tienden a ayudar menos a sus padres. En opinión de los padres, el tipo de trabajo que realizan, generalmente en la albañilería, difícilmente les alcanza para mantenerse a ellos mismos, de manera que en $85 \%$ del total de las personas mayores de esta comunidad no cuentan con hijos fuera de la localidad que los ayuden económicamente. En San Miguel y Mesón Viejo este porcentaje es de 33 y 15\%, respectivamente. Estas diferencias en la ayuda de los hijos migrantes parecen tener que ver con las condiciones en que emigran.

En Mesón Viejo, las condiciones de vida de los hijos fueron mejores. La época de auge de la papa permitió mantener a los hijos en la escuela e invertir en una mayor educación. Si bien no todos los hijos alcanzaron a terminar la primaria, en muchos hogares se logró que continuaran la secundaria (a pesar de que no se cuenta con escuelas de ese nivel en la comunidad) y no pocos, inclusive, terminaron estudios superiores como normalista, enfermería y agronomía. En esta comunidad se da una fuerte valoración a la formación escolar de los hijos. En San Miguel, donde también pudieron obtener ganancias de la agricultura comercial, se encuentran más hogares con esta tendencia, mien- 
tras que en San Mateo la expectativa de los padres hacia la escolarización de sus hijos se restringió a que aprendieran a leer y a sumar.

Los hijos que residen en la localidad pero fuera del hogar son los que menos ayuda económica brindan a sus padres. Las mujeres casadas prácticamente están excluidas de esta obligación, pero prevalece la justificación para que tanto las parejas de sus hijas como los hijos varones no aporten ingresos, por el hecho de "ser pobres". La ayuda de los hijos varones principalmente es en la explotación de la parcela de los padres, sobre todo cuando no reside con ellos otro pariente que también trabaje y dependa de la parcela.

Algunos productores de San Miguel y Mesón Viejo, mayores de 60 años de edad, continúan explotando sus tierras ejidales y privadas y, en algunos casos, parcelas arrendadas en comunidades con mejores tierras. Estos agricultores cuentan con la ayuda de hijos residentes en la localidad pero bajo la dirección de los padres (ya que administran y comercializan el producto de la explotación agrícola), por lo que perciben que son ellos (los padres) los que continúan ayudando a sus hijos.

Las estrategias para sostenerse que siguen los mayores de 60 años cuando no tienen hijos residiendo con ellos incluyen:

a) raspa y venta de pulque, así como otros productos misceláneos que les permiten alimentarse de estrictamente el mínimo para subsistir,

b) sembrar a medias la tierra con otros productores, participando en las labores agrícolas y con su tierra, a cambio de dinero en efectivo para los gastos de semilla y otros insumos. De este trabajo se obtiene maíz y frijol; y,

c) se recurre al trabajo asalariado en la tierra de otros (a lo que denominan "alquilarse"), lo cual ocurre tanto en los hombres como en las mujeres y sobre todo entre las familias más pobres.

Para ahondar en las condiciones en que los hijos apoyan a sus padres en la vejez, en el cuestionario aplicado a las personas mayores de 60 años se incluyó una tabla de hijos nacidos vivos, que indagaba sobre la ayuda económica que cada uno de ellos brindaba a sus padres. Esta ayuda es la aportación o el envío de dinero, aunque puede ser con una frecuencia muy variada.

En Temascaltepec, las personas mayores cuentan con la ayuda de 2 a 3 hijos en promedio. Las diferencias en este promedio son muy acentuadas entre comunidades. Mientras que en Mesón 
Viejo se cuenta con más de cuatro hijos, en San Miguel el promedio es de 2.3 y en San Mateo de 1.5. Si bien estas diferencias resultan en parte del número de hijos nacidos vivos que se tuvieron y del éxito de su sobrevivencia, el cual es superior en Mesón Viejo, son principalmente explicadas por la mayor participación de los hijos que residen fuera de la localidad. Así, mientras en Mesón casi $60 \%$ ayuda a sus padres, en San Mateo es menos de $18 \%$. En San Miguel también reciben ayuda de $\mathbf{6 0 \%}$ de los hijos migrantes, pero cuentan con un número inferior de ellos residiendo fuera de la localidad. En San Mateo casi la mitad de los hijos que brindan ayuda a sus padres son los hijos residentes en el hogar; el otro $40 \%$ lo constituyen hijos residentes en la localidad fuera del hogar.

En general, en las tres comunidades se observa que el matrimonio de los hijos varones representa un obstáculo para continuar apoyando a los padres. De esta manera, sólo un $44.8 \%$ de los hijos casados o unidos da ayuda económica a los padres, mientras que en el caso de los hijos solteros esta proporción es de 60.0 por ciento.

Si bien no sabemos cuál es la importancia real de la ayuda que brindan los hijos para la manutención de los viejos que la reciben, destaca el hecho de que poco más de una quinta parte de estas parejas o individuos no reciben ayuda económica por parte de ningún hijo en la región. En San Mateo es de 30\% de las familias de los viejos, en San Miguel de 22 y en Mesón de 13 por ciento. Lo anterior muestra que, si bien no es lo predominante, en las tres comunidades, a mayor pobreza mayor proporción de mayores que no cuentan con apoyo de sus hijos durante la vejez. Podría plantearse que la menor ayuda de los hijos es la causa de la pobreza de los padres, sin embargo, las historias de vida de los entrevistados y el desarrollo de sus unidades de producción, sugiere que la base de la desigualdad actual se encuentra en un acceso diferenciado a los recursos productivos desde los inicios de las unidades de producción, al igual que a diferentes relaciones ccn los grupos de poder. Al parecer, el mismo desarrollo económico y social de las comunidades y al interior de ellas, de algunos productores, determinó el "éxito" de la inversión en los hijos como seguridad económica en la vejez.

Algunas características socioeconómicas de los hogares de los viejos

En los cuadros 4 y 5 se muestran algunos indicadores de las condiciones de vida de los hogares con y sin personas residentes de 60 años o más. Puede apreciarse que tanto en San Mateo como en 
CUADRO 3

Promedios de número de hijos vivos y de los que ayudan económicamente a los padres, según localidad de residencia (de personas de 60 años o más)

\begin{tabular}{lrrrrrrrrr}
\hline & $H N V$ & HVA & HRH & HRL & HRFL & HA & HRA & HRLA & HRFLA \\
\hline San Mateo & 8.7 & 5.0 & 1.2 & 2.2 & 1.7 & 1.5 & 0.7 & 0.6 & 0.3 \\
San Miguel & 8.0 & 5.5 & 0.9 & 1.0 & 3.5 & 2.3 & 0.4 & 0.1 & 1.8 \\
Mesón Viejo & 10.2 & 8.6 & 1.6 & 1.6 & 5.4 & 4.3 & 0.9 & 0.3 & 3.2 \\
Temascaltepec & 8.6 & 5.9 & 1.1 & 1.4 & 3.3 & 2.5 & 0.6 & 0.3 & 1.6 \\
\hline
\end{tabular}

Fuente: estudio "Determinantes del Uso de Métodos anticonceptivos en las Áreas Rurales". Cuestionario para personas de 60 años o más

Promedios de:

HNV $=$ hijos nacidos vivos.

HVA $=$ hijos vivos al momento de la entrevista.

$\mathrm{HRH}=$ hijos residentes en el hogar.

HRL $=$ hijos residentes en la localidad.

HRFL $=$ hijos residentes fuera de la localidad.

$\mathrm{HA}=$ hijos que ayudan económicamente a sus padres.

HRA $=$ hijos residentes en el hogar que ayudan.

HRLA = hijos residentes en la localidad que ayudan.

HRFLA = hijos residentes fuera de la localidad que ayudan.

San Miguel, los hogares de los viejos presentan condiciones similares o inferiores que los hogares donde no hay viejos. En Mesón Viejo, por el contrario, los hogares de los viejos presentan características muy superiores a los del resto de los hogares de la comunidad. Muchas de las familias de viejos en esta comunidad lograron mejorar sus condiciones de vida con los rendimientos de la explotación agrícola, por lo que, si bien el éxito de los hijos para apoyar a sus padres pudo haber influido, es más probable que haya resultado de los logros económicos de su unidad productiva independientemente de los hijos.

Preferencias sobre el tamaño de la familia de la población de 60 años o más

En la zona de estudio se ha iniciado una disminución de los niveles de la fecundidad como resultado de la expansión del uso de métodos anticonceptivos. Las mujeres en edad fértil, unidas o casadas, que al momento de la entrevista habían hecho uso de los métodos anticonceptivos alcanzaba $43 \%$ de todas las mujeres unidas de la región.

Entre las comunidades se observan diferencias sustanciales. En Mesón Viejo, $42 \%$ de las mujeres entrevistadas eran usuarias activas de un método anticonceptivo en el momento de la entre- 
CUADRO 4

Características socioeconómicas de los hogares sin personas de 60 años o más

\begin{tabular}{lrrrr}
\hline & & \multicolumn{3}{c}{ Mesón } \\
& Sn. Mateo & Sn. Miguel & Viejo & Total \\
\hline Tiene parcela & 89.8 & 91.8 & 67.3 & 86.9 \\
Una ha. de cultivo o menos & 77.4 & 55.4 & 76.5 & 70.2 \\
Uso de tractor & 0.7 & 11.8 & 8.6 & 5.6 \\
Uso de fertilizantes químicos & 94.7 & 81.4 & $\mathbf{9 1 . 4}$ & 89.5 \\
Uso de insecticidas & 2.0 & 31.4 & 85.7 & 22.6 \\
Material pisos: tierra & 80.2 & 42.7 & 32.7 & 60.2 \\
Material paredes: adobe y tabique & 41.3 & 60.0 & 75.0 & 52.9 \\
Material techo: teja y concreto & 1.2 & 8.2 & 40.4 & 9.7 \\
Agua entubada & 12.6 & 34.5 & 67.3 & 28.6 \\
Luz eléctrica & 45.5 & 79.1 & 80.8 & 62.3 \\
1 a 2 cuartos para dormir & 77.8 & 62.8 & 44.2 & 67.4 \\
Sin drenaje & 26.3 & 68.2 & 53.8 & 44.7 \\
Combustible cocina: leña & 97.0 & 94.5 & 76.9 & 93.0 \\
Tiene radio & 59.9 & 68.2 & 61.5 & 62.9 \\
Tiene TV & 4.8 & 14.5 & 7.7 & 8.5 \\
Tiene refrigerador & 0.6 & 1.8 & $\mathbf{9 . 6}$ & 2.4 \\
Tiene automóvil & 2.4 & 1.8 & 7.7 & 3.0 \\
\hline
\end{tabular}

Fuente: estudio "Determinantes del Uso de Métodos Anticonceptivos en las Áreas Rurales”. Cuestionario de Hogar.

vista y $64 \%$ había hecho alguna vez uso de ellos. En contraste, en San Mateo, sólo $18 \%$ era usuaria y menos de $30 \%$ los había usado alguna vez. San Miguel presenta la situación intermedia con 32\% de uso y $50 \%$ que alguna vez usó anticonceptivos, entre las mujeres unidas en edad fértil.

El tema de la anticoncepción y de la reducción del número de hijos era conocido por las personas mayores, principalmente por haber oído de ellos a otros parientes o en los medios de comunicación. La actitud generalizada de la población mayor es a favor de la reducción del tamaño de la familia. En lo que existen diferencias es en el temor por el daño a la salud que puedan causar los medios modernos de anticoncepción y en menor proporción a ir en contra de la "voluntad de Dios". Estos temores se declararon principalmente en San Mateo y San Miguel. En Mesón Viejo, la actitud es positiva a la anticoncepción e inclusive hubo quien respondió que le avergonzaba haber tenido tantos hijos.

La opinión de las mujeres sobre la conveniencia de controlar la fecundidad y reducir el tamaño de la familia se fundamenta 
CUADRO 5

Características socioeconómicas de los hogares con personas de más de 60 años o más

\begin{tabular}{lrrrr}
\hline & & & Mesón & \\
& Sn. Mateo Sn. Miguel & Viejo & Total \\
\hline Tiene parcela & 96.4 & 94.5 & 94.7 & 95.1 \\
Una ha. de cultivo o menos & 88.9 & 51.9 & 27.8 & $\mathbf{5 7 . 7}$ \\
Uso de tractor & 0.0 & 7.7 & 22.2 & 8.2 \\
Uso de fertilizantes químicos & 92.6 & 84.6 & 94.4 & 88.7 \\
Uso de insecticidas & $\mathbf{0 . 0}$ & 37.3 & $\mathbf{8 8 . 9}$ & 36.5 \\
& & & & \\
Material piso: tierra & 82.1 & 56.4 & 0.0 & 52.9 \\
Material paredes: adobe y tabique & $\mathbf{4 6 . 4}$ & 69.1 & 94.7 & 67.6 \\
Material techo: teja y concreto & 3.6 & 10.9 & 47.4 & 15.7 \\
Agua entubada & 21.4 & 32.7 & 89.5 & 40.2 \\
Luz eléctrica & 42.9 & 81.8 & 100.0 & 74.5 \\
1 a 2 cuartos para dormir & 71.4 & 65.4 & 15.8 & 57.9 \\
Sin drenaje & 35.7 & 70.9 & 15.8 & 51.0 \\
Combustible cocina: leña & 100.0 & 94.5 & 57.9 & 89.2 \\
Tiene radio & 39.3 & 61.8 & 68.4 & $\mathbf{5 6 . 9}$ \\
Tiene TV & 3.6 & 12.7 & 10.5 & $\mathbf{9 . 8}$ \\
Tiene refrigerador & $\mathbf{0 . 0}$ & 1.8 & 26.3 & $\mathbf{5 . 9}$ \\
Tiene automóvil & $\mathbf{0 . 0}$ & 3.6 & 10.5 & 3.9 \\
\hline
\end{tabular}

Fuente: estudio "Determinantes del Uso de Métodos Anticonceptivos en las Áreas Rurales". Cuestionario de Hogar.

principalmente, en tres tipos de costos: el costo económico que representó la manutención y el cuidado de los hijos; el costo emocional que implicó su crianza y en cierta forma la reproducción de la pobreza y el costo en salud derivado de los embarazos y partos.

\section{a) Costos económicos}

El costo económico de los hijos se refiere esencialmente a la dificultad que significó alimentarlos día con día y curarlos cuando se enfermaban.

El gasto en educación fue prácticamente nulo, porque no se enviaba a los niños a la escuela, en parte porque no se contaba con ese servicio en la comunidad y en parte porque era más importante que ayudaran en las labores productivas de la unidad doméstica. Por otro lado, se gastaba poco en ropa o calzado. Los alimentos que se consumían eran menos variados que en la actualidad. Sin embargo, a pesar de que en términos relativos se gastaba poco en 
los hijos, para ellas la dificultad principal radicaba en que no podían asegurar la comida diaria. Las mujeres tenían que trabajar la parcela propia y la de otros, y los hombres salir de la comunidad a trabajar en las ciudades para obtener el alimento del día.

Si uno toma cosas (anticonceptivos ) se puede enfermar, pero también como está la vida de dura tampoco es bueno tener tanta familia porque sufre uno para darles de comer.

Ahora ya se puede tener familias más chicas, como de tres hijos está bien, por las dificultades que se tiene para mantenerlos o darles estudio. No me gusta pensar que mis hijos sufran lo que yo sufrí para darles de comer a tantos o por no tener la forma de curarlos cuando se enfermaron; si le digo que yo sufrí mucho con mi esposo porque el no nos dio más que malos tratos y ni media ayuda cuando lo necesitamos.

Si se enfermaban se les curaba con remedios caseros, porque era difícil trasladarlos con un curandero que no fuese de la comunidad o con un médico. Casi todas las mujeres sufrieron la muerte de 3 o 4 hijos y en muchos casos se piensa que la muerte ocurrió por no haber contado con los medios económicos para llevarlos a curar.

También se percibe que las demandas actuales de los hijos se han incrementado, debido principalmente, a su mayor escolarización, por lo que las parejas jóvenes deben reducir el tamaño de la familia.

El trabajo asalariado y principalmente la emigración desde temprana edad era, desde entonces, una estrategia común, no sólo para que la familia obtuviera ingresos monetarios, sino también para mejorar las condiciones de vida de los jóvenes migrantes, para que pudieran comprar lo que ellos demandaban como ropa y zapatos, además de su alimentación.

Estas personas declaran, en su mayoría, que de haber contado con medios para evitar el nacimiento de un hijo, no habrían tenido tantos, siendo la principal causa, las dificultades que enfrentaron para darles de comer. Así, es posible ubicar como la principal causa para estar de acuerdo con el control de los nacimientos el alto costo que significa su sobrevivencia, a pesar de que ésta haya sido, en su experiencia, a un nivel de infrasubsistencia.

\section{b) Costo emocional}

Otro de los aspectos que casi todas las mujeres resaltaron fueron los problemas que implicó la convivencia con el marido, generalmente alcohólico, que ejerció una fuerte violencia hacia ellas y 
hacia los hijos. De esa forma, más hijos significaban para la mujer más problemas y más trabajo...

La vida de la mujer es puro trabajo y sufrimiento, lo cual se empeora con más hijos.

Unido a ello, existe un fuerte pesimismo con respecto al futuro de estos hijos, lo que también refuerza la ventaja de reducir el número de nacimientos.

Se maneja el concepto de que los hijos pueden resultar "buenos o malos" dependiendo de si responden o no a las expectativas de comportamiento y solidaridad hacia sus padres. Cuando se teme que los hijos "no salgan buenos", se teme que sean alcohólicos, que no trabajen o que desaparezcan de la vida de éstos.

Yo sí esperaba que siquiera me vieran, pero ya ve, de siete que tuve sólo conté con dos y ahora mis hijos ni siquiera me ven ni me ayudan.

...uno de mis hijos me salió borracho y el otro hasta ha llegado a golpearme; a Julio yo sí lo aborrezco, ya van dos veces que me golpea, yo ni aprecio le tengo.

Gracias a Dios todos me resultaron buenos, ya ve, muchas mujeres tienen hartos y todos las olvidan.

En ocasiones se tiene en el hogar a la mujer abandonada por el hijo, o a los hijos de la hija que "fracasa" (éstas son las madres solteras), implicando no sólo que los hijos no retribuyan, sino que además sigan generando gastos a sus padres.

En este sentido, el costo emocional se refiere esencialmente a tres aspectos: los hijos hacen más vulnerables a las mujeres respecto a la violencia y maltrato de la pareja. La maternidad y la crianza de los hijos refuerza su subordinación al marido, lo que en el plano económico se manifiesta de dos maneras aparentemente contradictorias; por un lado, la hace más dependiente del aporte económico de su pareja y por otro, la obliga a encontrar los mecanismos que permitan la sobrevivencia de sus hijos. En general, muchas de las mujeres entrevistadas perciben una vida tormentosa junto a la pareja, debido principalmente a la falta de "responsabilidad" por parte del hombre para el trabajo y sostenimiento de los hijos, lo que las obligó a duplicar o triplicar las jornadas de trabajo, para atender las demandas domésticas y económicas de la familia, lo cual se agudizaba con los nacimientos continuos de los hijos. Además, la maternidad y la sexualidad se vivía con una gran frustración y rencor hacia el marido, por lo que se deseaba que "Dios" o la "suerte" no les diera más hijos. 
c) Costos en salud

Otro tipo de costos señalados por los entrevistados, se refieren a los de la salud de la madre. Para las mujeres, los embarazos significaban un riesgo a su salud, un riesgo de muerte, por lo que también hubieran preferido tener menos hijos. Inclusive, el deterioro de su estado de salud actual lo asocian a tantos embarazos, tanto trabajo y tantos hijos, por lo que piensan que ahora la gente puede vivir mejor si reducen el número de nacimientos.

En relación con las razones de salud de las madres para estar a favor de un menor tamaño de familia, algunas mujeres plantearon el miedo a la muerte que les ocasionaban los partos:

Uno se las veía sola (en el parto) y no era como ahora que está al pendiente de las mujeres un médico, uno se veía con un pie en la tierra y otro en la sepultura.

La preferencia por un tamaño menor de familia se manifestó, en algunos casos, en el ejercicio de mecanismos concretos para evitar los nacimientos, como haber dejado de tener relaciones sexuales con el esposo para ya no tener más familia.

Yo por eso ya no quise meterme con el señor (su esposo) porque la vida ya estaba muy pesada y más hijos más dificultades... sólo una sabe lo que se sufre teniendo hijos.

\section{A manera de conclusión}

Los hijos otorgan a sus padres asistencia instrumental que incluye la ayuda en el hogar, la ayuda financiera y práctica y la ayuda en la vejez. Sin embargo, los datos analizados sugieren que una familia numerosa no implica una mejoría en las condiciones de vida de los padres. En cuanto a la ayuda en la vejez, pareciera también que muchos hijos no necesariamente conllevan a una mayor seguridad económica.

Para los hombres y mujeres de 60 años o más, son mayores las desventajas percibidas que las ventajas de tener muchos hijos. Y uno podría leer en sus declaraciones el énfasis dado a los problemas de la pobreza que implica tener hijos.

Inclusive durante la última etapa de la vida de los individuos, los más pobres son los que más necesidad tienen de continuar manteniéndose a sí mismos hasta prácticamente la muerte, en precarias condiciones económicas. 
Parecería entonces que la explicación del cambio en los niveles de la fecundidad que ha ocurrido en estas comunidades se ubica más al nivel de los costos de la reproducción que de sus beneficios económicos. Sin ser concluyente, el trabajo sugiere que aspectos relativos a la condición de género y pobreza deben ser considerados en la explicación de la reducción del número de hijos.

El inicio de una nueva pareja sigue arreglos sociales en los que se relaciona la solidaridad de los adultos hacia una pareja más joven con mecanismos para mantener la ayuda de un hijo una vez que se casa. Incluso la salida de esa pareja del hogar paterno se relaciona con la llegada de otros hijos que inician su matrimonio.

Este proceso se sigue a lo largo del ciclo de vida de los padres. Algunos de los hijos residirá con ellos al final de su vida, y él será quien herede los medios de producción a su muerte. Sin embargo, no siempre resulta atractivo para los hijos permanecer en la comunidad y desarrollar las actividades de sus padres. En estos casos, el largo proceso de "capacitación" de la fuerza de trabajo que se inicia desde una temprana edad, proceso que permite acrecentar el uso de la fuerza de trabajo familiar y concretar su uso "flexible", no se logra consolidar el mecanismo de solidaridad de la generación más joven con la generación más vieja de acuerdo con la costumbre de la comunidad.

Aunque la mitad de los hogares donde viven personas mayores de 60 años se conforma por estructuras familiares extensas, en la otra mitad estas viven solas o con sus parejas. Además, en una alta proporción, los individuos en la tercera edad que reside en hogares extensos son reconocidos como jefes económicos del hogar. Esto muestra que el proceso de reproducción familiar no asegura el apoyo económico de los hijos hacia sus padres durante la vejez, que permita a las personas dejar de trabajar durante la última etapa de su vida.

Esto no implica, sin embargo, que los hijos no jueguen un importante rol económico o de asistencia instrumental durante la vida. Al alcanzar la edad para tener una mejor oportunidad de asalariarse, se considera la etapa en que los hijos más ayudan al hogar. Esta posibilidad se maximiza con su emigración a las zonas urbanas. De nueva cuenta, los lazos de solidaridad familiar son los que mantiene este mecanismo de reproducción; la "obligación" de apoyo a distancia persiste hasta el matrimonio o la llegada de hijos en la nueva pareja. Estas formas de apoyo a distancia en realidad parecen depender de la "buena voluntad" de los hijos, y no es poco frecuente que se les describa como "salió bueno" o "salió malo" por sus padres. Futuros trabajos deberán ahondar en los mecanismos que debilitan los elementos de solidaridad familiar. 


\section{Bibliografía}

Cain, Mead (1981), "Risk and insurance: perspectives on fertility and inequality in rural India and Bangladesh", Working Papers, Nueva York, Population Council.

Caldwell, J. (1983), "Direct economic costs and benefits of children", en Bulatao y Lee (eds.), Determinants of fertility in developing countries, Nueva York, Academic Press.

Fawcett, J. (1983), "Perceptions of the value of children: satisfactions and costs", en Bulatao y Lee (eds.), Determinants of ifertility in developing countries, Nueva York, Academic Press.

Hull, T. (1983), "Cultural influences on fertility decision styles", en Bulatao y Lee (eds.), Determinants of fertility in developing countries, Nueva York, Academic Press.

McNicoll, G. (1983), "The nature of institutional and community effects on demographic behavior: an overview", en Working Papers, Nueva York, Population Council.

Zúñiga E., D. Hernández, Ma. E. Rojano y C. Santos (1988), "Determinantes del uso de métodos anticonceptivos en el área rural de México: estudio comparativo micro-regional", México (mimeo.). 